\title{
DISTRIBUTION OF PHENOLIC COMPOUNDS, PECTINS AND HEMICELLULOSES IN MATURE PIT MEMBRANES AND ITS VARIATION BETWEEN PIT TYPES IN ENGLISH OAK XYLEM (QUERCUS ROBUR)
}

\author{
Jong Sik Kim and Geoffrey Daniel* \\ Wood Science, Department of Forest Products, Swedish University of Agricultural Sciences, \\ P.O. Box 7008, 75007 Uppsala, Sweden \\ *Corresponding author; e-mail: geoffrey.daniel@slu.se
}

\begin{abstract}
Although there is considerable information on the chemistry of bordered intervessel pit membranes, little is known on the pit membrane chemistry of other pit types in hardwoods. This study investigated distribution of phenolic compounds, pectins and hemicelluloses in different mature pit membranes of English oak xylem using transmission electron microscopy coupled with cytochemistry and immunocytochemistry. Mature bordered intertracheid (vasicentric)- and tracheid-vessel pits showed presence of xyloglucan and heteromannan (hemicelluloses) epitopes across the pit membrane (except for the annulus regions) with differences in amounts of epitopes between earlywood (EW) and latewood (LW). In contrast, pectin epitopes were detected only in the annulus regions of pit membranes. Unlike bordered pits, half-bordered (tracheary-parenchyma pits) and simple (parenchyma pits) pit membranes were rich in pectin epitopes but lacked heteromannan epitopes, indicating difference in pit membrane chemistry between pit types. Distribution of phenolic compounds also differed between pit types and between EW and LW. LW also showed great variations in distribution of phenolic compounds between vessels. Together, this study demonstrates that there are great variations in pit membrane chemistry between pit types and between EW and LW in English oak xylem.
\end{abstract}

Keywords: Bordered pit, half-bordered pit, immunocytochemistry, heteromannan, non-cellulosic polysaccharide, pit membrane chemistry, simple pit, xyloglucan.

\section{INTRODUCTION}

Pits have received considerable attention due to their importance in the regulation of water flow within the xylem of living trees. In particular, much attention has been paid to pit membranes in relation to safety and efficiency of the hydraulic system within xylem (Zwieniecki et al. 2001; Choat et al.2008; Nardini et al.2011; van Doorn et al. 2011; Schenk et al. 2015; Li et al.2016). With respect to hardwoods, most attention has been paid to pit membranes of intervessel pits since their water transport is performed 
mostly by vessels. Pit membranes of other pit types, such as those between vessels and parenchyma cells, between vessels and imperforated cells (i.e. tracheids, fiber-tracheids, libriform fibers) and between imperforate cells have rarely been studied in hardwoods (Sano \& Jansen 2006; Sano et al.2011). At the same time, the function of pit membranes in hardwood species that have mostly solitary vessels (i.e. relatively few intervessel pits) is poorly understood. In oak xylem, vessels are predominantly solitary (Schweingruber 1990; InsideWood 2004-onwards). This suggests the potential importance of other pit types to facilitate water conduction in oak xylem. However, very little information is available on the chemistry of pit membranes in oak.

In an effort to understand the chemistry of fully mature pit membranes in hardwoods, many cytochemical and immunocytochemical studies have been conducted in several hardwood species. However, the chemistry of pit membranes in hardwoods remains unclear. In particular, it is under debate whether or not pectins are present in mature intervessel pit membranes (bordered pits) (Gortan et al. 2011; Nardini et al. 2011). While classical chemical staining using hydroxylamine-ferric chloride, ruthenium red and alcian blue showed presence of pectins in intervessel pit membranes of several hardwood species (Jansen et al. 2004; Gortan et al. 2011), immunocytochemical studies using antibodies revealed absence of pectin epitopes in the main structural part of intervessel pit membranes (Plavcová \& Hacke 2011; Plavcová et al. 2011; Kim \& Daniel 2013; Herbette et al. 2015). Pectin epitopes were only detected in the annulus of intervessel pit membranes. Since the hydrogel properties of pectins in bordered pit membranes are currently thought to be strongly correlated with the ion-mediated changes of xylem hydraulic conductance (i.e. swelling/shrinking dynamics of a porous pectic matrix depend on ionic concentrations in the xylem) in hardwoods, a question whether presence or absence of pectins in mature bordered pit membranes remains an important issue (Zwieniecki et al. 2001; Nardini et al. 2011; van Doorn et al. 2011). Pectin epitopes were also detected in vessel-ray (half-bordered pit), fiber-ray (simple pit), fiber (simple pit) and ray (simple pit) pit membranes of several hardwood species (Rioux et al. 1998; Plavcová \& Hacke 2011; Kim \& Daniel 2013). Little information is available on hemicelluloses in pit membranes of hardwoods. Immunocytochemical studies showed distribution of xyloglucan epitopes in mature intervessel pit membranes of grapevines and European aspen (Sun et al. 2011; Kim \& Daniel 2013). In contrast, xyloglucan epitopes were not detected in mature intervessel pit membranes of hybrid aspen and poplar using similar immunocytochemical approaches (Kim \& Daniel 2013; Herbette et al. 2015). No clear evidence for the presence of heteromannan and heteroxylan in mature intervessel pit membranes has been reported in hardwoods (Kim et al. 2012; Herbette et al. 2015). In contrast, heteroxylan epitopes were detected in mature vessel-ray pit membranes of hybrid aspen (Kim et al. 2012).

In this study, we investigated the microdistribution of non-cellulosic polysaccharides (pectins and hemicelluloses) in different pit membranes of English oak xylem. The chemistry of fully mature pit membranes: 1) between vasicentric tracheids (bordered pits); 2) between vasicentric tracheids and vessels (bordered pits); 3) between vasicentric tracheids and axial/ray parenchyma cells (half-bordered pits); 4) between vessels and axial/ray parenchyma cells (half-bordered pits); and 5) between two axial 
parenchyma cells (simple pits) was examined using immunocytochemistry combined with monoclonal antibodies that bind to galactan/arabinan (rhamnogalacturonan I, RG-I), homogalacturonan (HG), xyloglucan, heteroxylan and heteromannan epitopes. Distribution of phenolic compounds in pit membranes was also examined after $\mathrm{KMnO}_{4}$ staining.

\section{MATERIALS AND METHODS}

A small wood block was removed from a mature English oak tree (Quercus robur L.; c. $20 \mathrm{~cm}$ diameter at breast height) grown at a forest site near the campus of the Swedish University of Agricultural Sciences on September 16, 2014. Small sectors of the second and third growth ring (sapwood) from the cambial zone (i.e. fully developed growth ring) were cut from the wood block and fixed in a mixture of paraformaldehyde and glutaraldehyde (Kim \& Daniel 2014). Sectors were then embedded in LR White resin by conventional embedding procedures (Kim et al. 2012).

For transmission electron microscopy (TEM), transverse ultrathin sections (c. $90 \mathrm{~nm}$ ) were prepared from embedded blocks. To identify phenolic compounds, sections were stained with $1 \% \mathrm{KMnO}_{4}$. Immunogold labeling was conducted according to procedures described previously (Kim \& Daniel 2013). In brief, sections were incubated with either LM5 (1,4- $\beta$-galactan, Jones et al. 1997), LM6 (1,5- $\alpha$-arabinan, Willats et al. 1998), LM10/LM11 (un/low and low/highly substituted heteroxylan respectively, McCartney et al. 2005), LM15 (XXXG motif of xyloglucan, Marcus et al. 2008), LM19/LM20 (un/low and low/highly methyl-esterified homogalacturonan respectively, Verhertbruggen et al. 2009) or LM21 (heteromannan, Marcus et al. 2010) monoclonal antibodies (PlantProbes, Leeds, U.K.), followed by incubation with secondary antibody labeled with 10-nm colloidal gold particles (BBInternational, Cardiff, U.K.). Sections were examined using a Philips CM12 TEM (Philips, Eindhoven, Netherlands) after staining with uranyl acetate. No non-specific binding of secondary antibody (gold particles) was detected in control samples where the primary antibody was omitted (not shown).

\section{RESULTS}

Although presence of fiber-tracheids (having slit-like bordered pits) has been reported in oak xylem, all imperforate cells having bordered pits observed in this study were defined as vasicentric tracheids. Since English oak showed solitary vessel distribution in xylem, the presence of intervessel pits was unclear and therefore they were excluded in the study. Half-bordered pits were formed between tracheary elements (vasicentric tracheids and vessels) and axial/ray parenchyma cells in English oak xylem. Halfbordered pits associated with ray parenchyma cells were more frequent than pits associated with axial parenchyma cells in earlywood (EW). In contrast, latewood (LW) showed an opposite pattern. Although half-bordered pits between tracheary elements and axial parenchyma cells were mainly observed in this study, the types of parenchyma cells are not indicated in the text since structure and chemistry of half-bordered pit membranes were similar regardless of parenchyma cell type. Simple pits in English oak xylem were found in several cell types including fibers, ray parenchyma cells and 
Table 1. Distribution of phenolic compounds, pectin and hemicellulose epitopes in mature pit membranes of English oak xylem as reflected by $\mathrm{KMnO}_{4}$ staining and immunogold labeling.

Classification of earlywood (EW) and latewood (LW) indicates prominent differences in staining and labeling patterns between two anatomical regions. Definition of anatomical regions in pit membranes including membrane (main structural part of membrane), annulus and protective layer (PL) is shown in Figure 1. Intertracheid and tracheid-vessel pits (bordered pit); tracheid/vessel-parenchyma pits (half-bordered pit); parenchyma pits (simple pit).

\begin{tabular}{|c|c|c|c|c|c|c|c|c|c|}
\hline \multirow{2}{*}{ Pit type } & \multirow{2}{*}{$\frac{\text { Phenolic }}{\mathrm{KMnO}_{4}}$} & \multicolumn{2}{|c|}{ RG-1 } & \multicolumn{2}{|c|}{ HG } & \multirow{2}{*}{$\frac{\mathrm{XG}}{\mathrm{LM} 15}$} & \multirow{2}{*}{$\frac{\text { Mannan }}{\text { LM21 }}$} & \multicolumn{2}{|c|}{ Xylan } \\
\hline & & LM5 & LM6 & LM19 & LM20 & & & LM10 & LM11 \\
\hline \multicolumn{10}{|l|}{ Intertracheid pit } \\
\hline membrane & - & - & - & - & - & $\mathrm{EW}+$ & + & - & - \\
\hline annulus & + & - & + & + & + & $\begin{array}{c}\mathrm{LW} \mathrm{tr} /- \\
\mathrm{tr} /-\end{array}$ & $\mathrm{tr} /-$ & $\mathrm{tr} /-$ & $\operatorname{tr} /-$ \\
\hline \multicolumn{10}{|l|}{ Tracheid-vessel pit } \\
\hline membrane & $\begin{array}{c}\mathrm{EW}+ \\
\mathrm{LW} \mathrm{tr} /-\end{array}$ & - & - & - & - & $\begin{array}{c}\mathrm{EW}+ \\
\mathrm{LW} \mathrm{tr} /-\end{array}$ & + & - & - \\
\hline annulus & + & - & + & + & + & $\operatorname{tr} /-$ & $\operatorname{tr} /-$ & $\operatorname{tr} /-$ & $\operatorname{tr} /-$ \\
\hline \multicolumn{10}{|l|}{ Tracheid-parenchyma pit } \\
\hline membrane & $\operatorname{tr}$ & $+/-$ & + & + & + & - & - & - & - \\
\hline protective layer & - & - & + & - & + & $+/-$ & - & - & - \\
\hline \multicolumn{10}{|l|}{ Vessel-parenchyma pit } \\
\hline membrane & $\operatorname{tr}$ & - & + & + & + & - & - & - & - \\
\hline protective layer & - & - & + & - & + & $+/-$ & - & - & - \\
\hline Parenchyma pit membrane & + & + & + & + & + & $\operatorname{tr} /-$ & $\operatorname{tr} /-$ & - & $\operatorname{tr} /-$ \\
\hline
\end{tabular}

$+=$ positive staining /labeling; $\operatorname{tr}=$ trace staining/labeling; - = negative staining/labeling; $\mathrm{RG}-\mathrm{I}$ = rhamnogalacturonan $\mathrm{I} ; \mathrm{HG}=$ homogalacturonan; $\mathrm{XG}=$ xyloglucan.

axial parenchyma cells. However, in this study we focused only on simple pit connections between axial parenchyma cells. No notable formation of tyloses was detected in any of the cell types.

The overall distribution patterns of phenolic compounds and non-cellulosic polysaccharide epitopes in different mature pit membranes of English oak xylem are briefly summarized in Table 1. Annulus indicates the peripheral regions of bordered pit membranes (Fig. 1a). Pit membrane regions except for the annulus are defined as the main structural part of pit membranes (Fig. 1a). Protective layers (PL) indicate the part of parenchyma cell wall or the amorphous layer adjacent to half-bordered pit membranes (Fig. 1c, d). Protective layer in Table 1 indicates only the amorphous layer adjacent to half-bordered pit membranes. The possibility for changes in membrane chemistry in pit membranes associated with parenchyma cells is still likely since parenchyma cells are living cells even in mature xylem.

\section{Detection of phenolic compounds}

Figure 1 shows different pit membranes in EW after staining with $\mathrm{KMnO}_{4}$ for phenolic compounds. No notable $\mathrm{KMnO}_{4}$ staining was detected in bordered pit membranes between vasicentric tracheids (T-T, intertracheid pits, Fig. 1a) compared to controls 

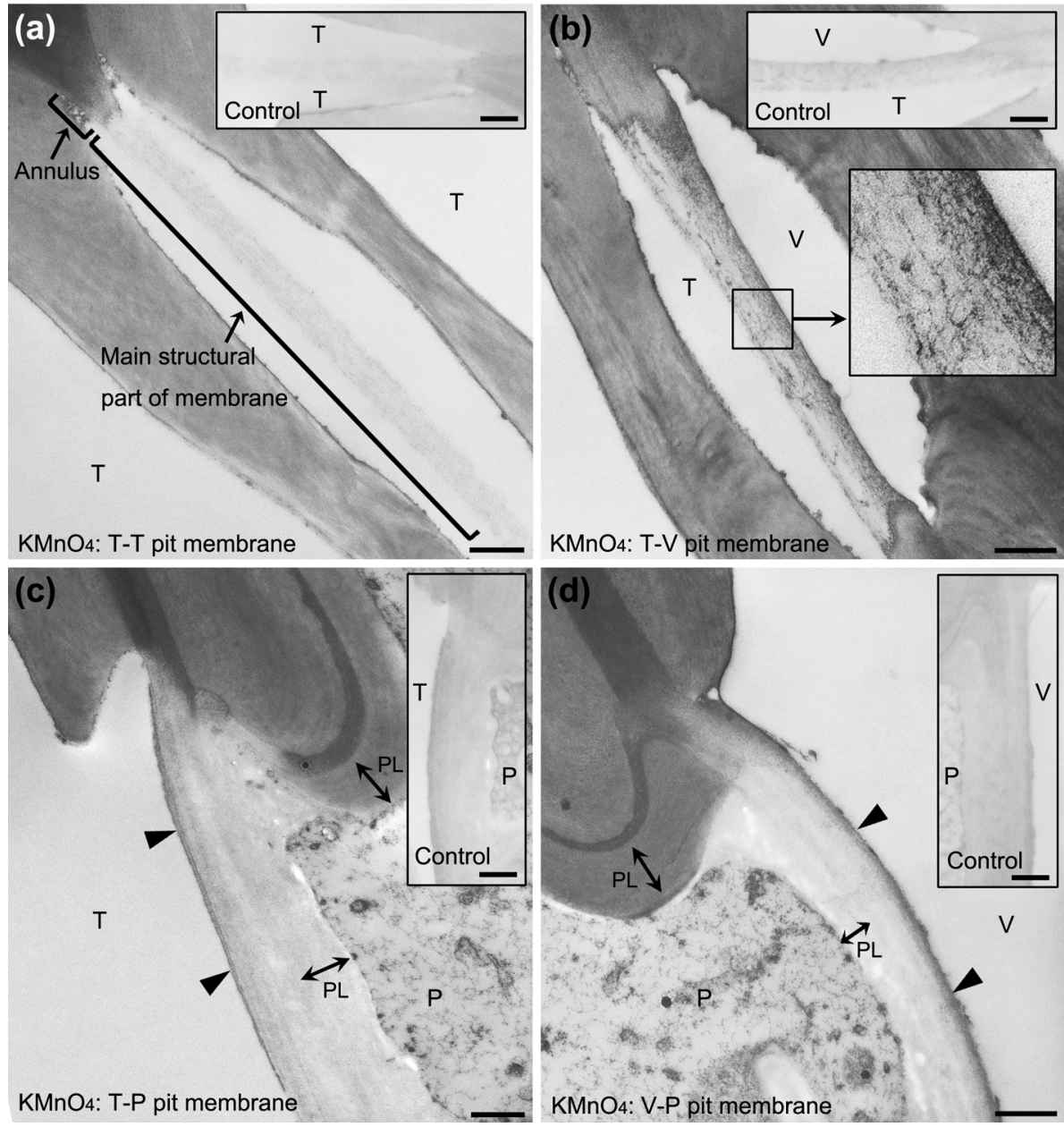

Figure 1. Pit membranes of English oak earlywood stained with $\mathrm{KMnO}_{4}$. Insets in a-d indicate controls without staining. - (a) Intertracheid pit membrane (T-T) showing almost negative staining. - (b) Tracheid-vessel pit membrane (T-V) showing positive staining and fibrillar network (bottom inset). - (c, d) Tracheary-parenchyma pit membranes (T-P/V-P) showing some positive staining on the surface facing tracheid and vessel lumina (arrowheads). Note development of protective layers (PL) in pit regions and inside parenchyma cell walls with differences in lignification. - Scale bars $=500 \mathrm{~nm}$.

(i.e. without $\mathrm{KMnO}_{4}$ staining, inset in Fig. 1a). In contrast, bordered pit membranes between vasicentric tracheids and vessels (T-V, tracheid-vessel pits, Fig. 1b) showed positive $\mathrm{KMnO}_{4}$ staining compared to control (upper inset in Fig. 1b). Tracheid-vessel pit membranes generally showed a fibrillar network with compact structure on the vessel side (lower inset in Fig. 1b). Half-bordered pit membranes between tracheary elements (vasicentric tracheids and vessels) and axial/ray parenchyma cells (T-P or V-P, tracheary-parenchyma pits) showed some positive $\mathrm{KMnO}_{4}$ staining on the surface fac- 
ing the tracheid and vessel lumina (arrowheads in Fig. 1c, d) compared with controls (insets in Fig. 1c, d). No notable $\mathrm{KMnO}_{4}$ staining was detected in protective layers (PL) of tracheary-parenchyma pit membranes, whereas protective layers developed inside parenchyma cell walls showed strong lignification (Fig. 1c, d). Some positive $\mathrm{KMnO}_{4}$ staining was detected in simple pit membranes between axial parenchyma cells (AP-AP, parenchyma pit, Fig. 8a) compared with control (see inset in Fig. 8a).
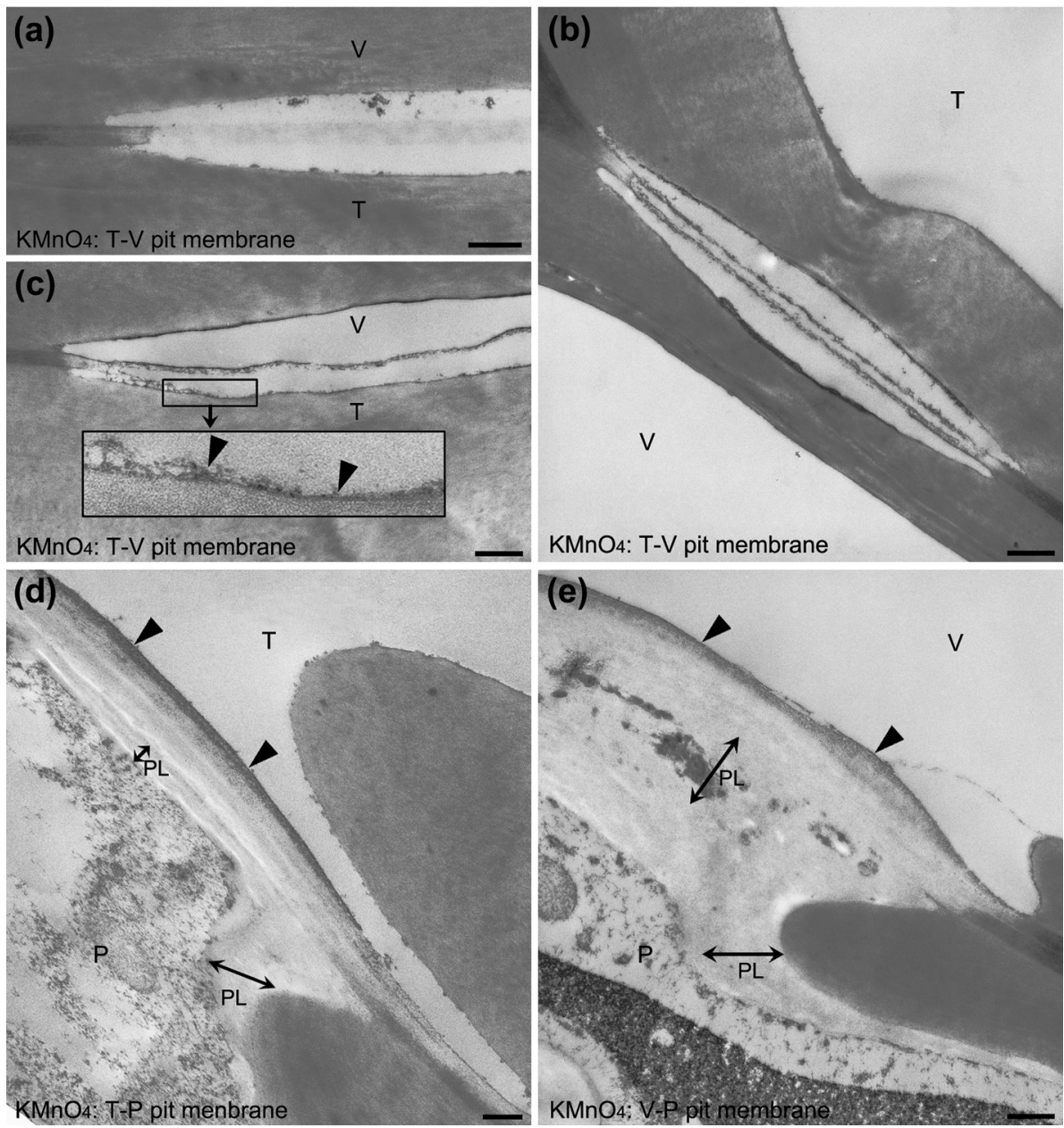

Figure 2. Pit membranes of English oak latewood stained with $\mathrm{KMnO}_{4} \cdot-(\mathrm{a}-\mathrm{c})$ Tracheid-vessel pit membrane (T-V) showing negative (a) and positive (b, c) staining. Note almost no content in the middle of membrane (b) and a single layer of membrane (c) attaching one side of the membrane to the pit border (arrowheads, inset in c). - (d, e) Tracheary-parenchyma pit membranes (T-P/V-P) showing some positive staining of the surface facing tracheid and vessel lumina (arrowheads). Note almost negative phenolic staining in both protective layers (PL) developed in pit regions and inside parenchyma cell walls (i.e. differ from EW; Fig. 1c, d). - Scale bars $=500 \mathrm{~nm}$. 
Like EW, $\mathrm{KMnO}_{4}$ staining was almost negative in intertracheid pit membranes of LW (not shown). In contrast, unlike EW tracheid-vessel pit membranes, LW showed two distinct patterns of $\mathrm{KMnO}_{4}$ staining; 1) negative staining like intertracheid pit membranes (Fig. 2a), and 2) positive staining with weak/or no intensity in the middle of pit membranes (Fig. 2b,c). The latter pit membranes frequently appeared as a single layer due to attachment of one side of the membrane to the pit border (arrowheads, inset
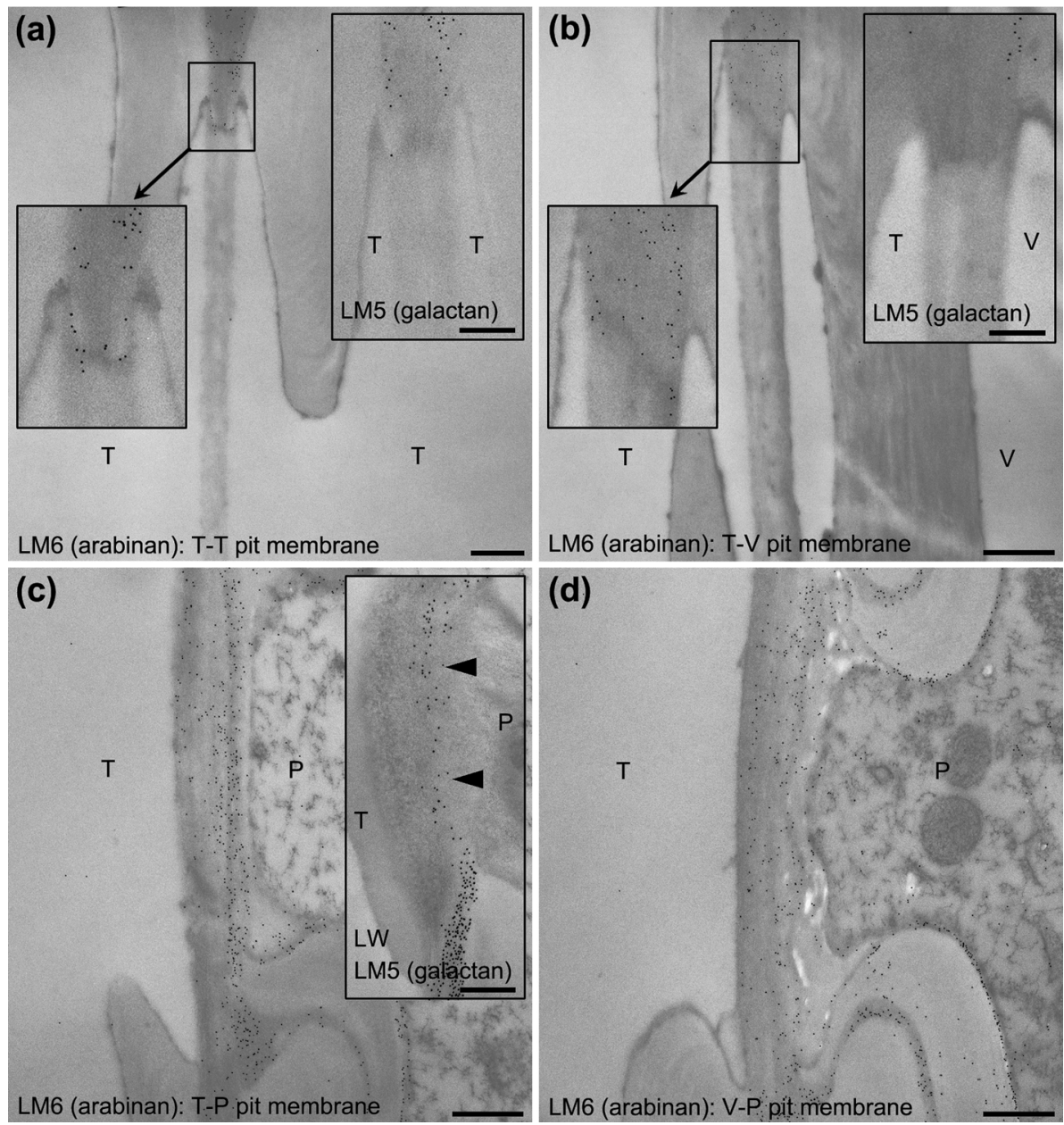

Figure 3. Localization of LM5 (galactan) and LM6 (arabinan) epitopes in pit membranes of English oak. All pits from earlywood except for the inset in c (from latewood, LW). - (a, b) Intertracheid (T-T, a) and tracheid-vessel (T-V, b) pits showing absence of LM5 (right insets in $\mathrm{a}, \mathrm{b})$ and LM6 (a, b) epitopes in the main structural part of pit membrane. Note LM6 epitopes in annulus of pit membranes (left insets in a, b). - (c, d) Tracheary-parenchyma pits (T-P/V-P) showing presence of LM6 epitopes across pit membranes. Note sparse LM5 epitopes in T-P pit membranes that were only detected in a few pits of LW (arrowheads, inset in c). - Scale bars $=$ $250 \mathrm{~nm}$ (insets in a, b), $500 \mathrm{~nm}$ (a-d). 

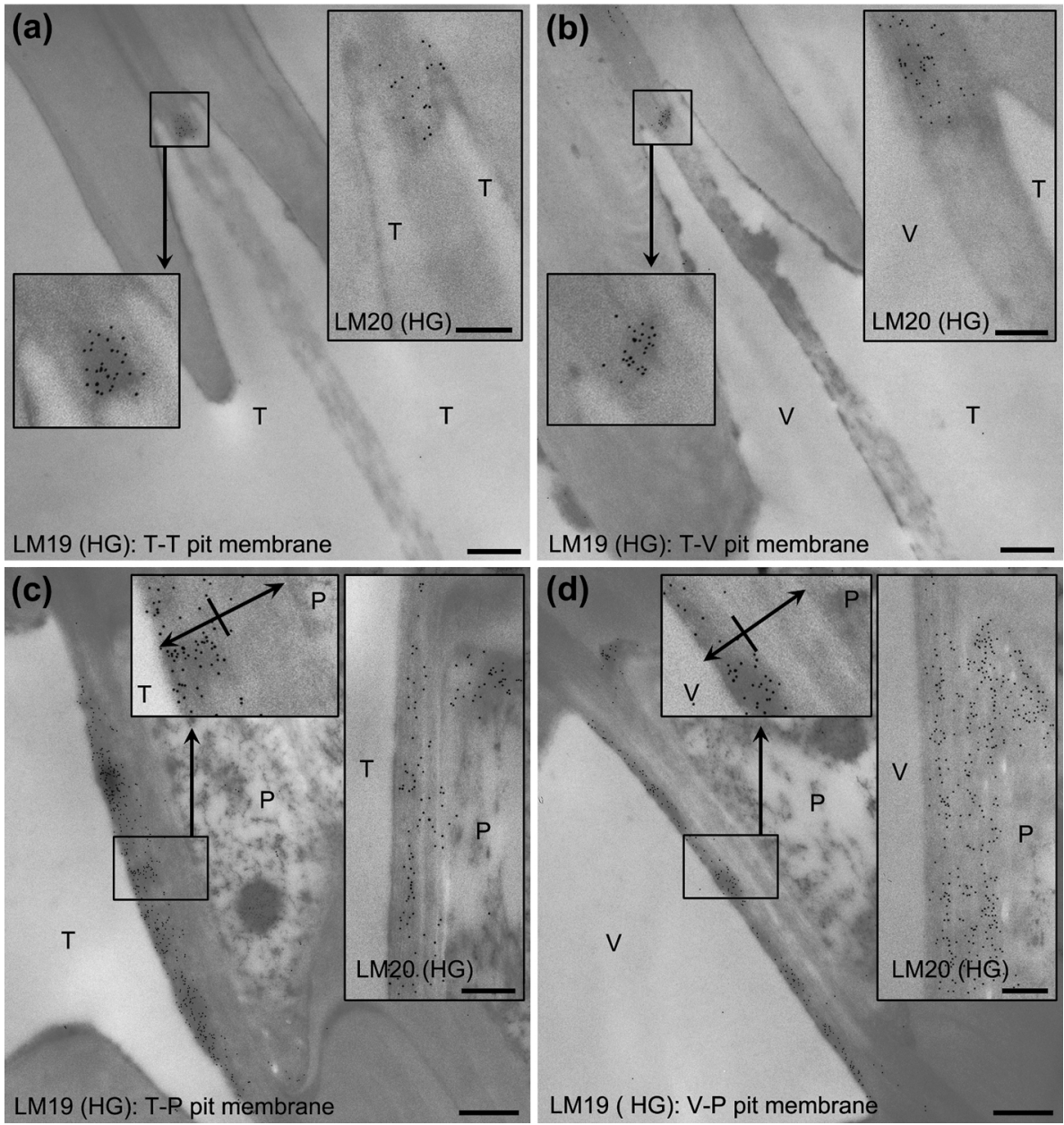

Figure 4. Localization of LM19 (low methyl-esterified homogalacturonan, HG) and LM20 (high methyl-esterified HG) epitopes in pit membranes of English oak. All pits from earlywood. - (a, b) Intertracheid (T-T, a) and tracheid-vessel (T-V, b) pits showing absence of LM19 $(a, b)$ and LM20 (right insets in a, b) epitopes in the main structural part of pit membrane. Note two epitopes in annulus of pit membranes (left insets in a, b).-(c, d) Tracheary-parenchyma pits (T-P/V-P) showing different distribution patterns between two antibodies; LM19 epitopes only present in narrow regions of pit membranes close to $\mathrm{T}$ and $\mathrm{V}$ lumina (c, $\mathrm{d}$ and left insets) and LM20 epitopes across pit membranes (right insets in c, d). - Scale bars $=250 \mathrm{~nm}$ (insets in $\mathrm{a}-\mathrm{d}), 500 \mathrm{~nm}(\mathrm{a}-\mathrm{d})$.

in Fig. 2c), probably aspiration of the pit membrane. All tracheid-vessel pits developed in the same vessel showed a single pattern of $\mathrm{KMnO}_{4}$ staining in pit membranes (i.e. either negative or positive). Some positive $\mathrm{KMnO}_{4}$ staining was detected in trachearyparenchyma pit membranes of LW but was restricted to the surface opposite the tracheid and vessel lumina similar to EW (arrowheads in Fig. 2d, e). Unlike EW, the protective 
layers developed inside parenchyma cell walls gave almost negative $\mathrm{KMnO}_{4}$ staining (Fig. 2d, e). Parenchyma pit membranes of $\mathrm{LW}$ showed positive $\mathrm{KMnO}_{4}$ staining similar to EW (not shown).

\section{Localization of pectin epitopes}

Localization patterns of pectin epitopes in pit membranes were similar between EW and LW. LM5 (galactan) and LM6 (arabinan) epitopes which indicate presence of rhamnogalacturonan I (RG-I) were not detected in the main structural part of intertracheid- and tracheid-vessel pit membranes (Fig. 3a, b and right insets). Epitopes were detected only in the annulus of pit membranes by LM6 (left insets in Fig. 3a, b). In tracheary-parenchyma pit membranes, abundant epitopes were detected by LM6 (Fig. 3c, d). LM 5 epitopes were generally absent in these pit membranes (not shown). The only exception was a few pits in LW that showed some LM5 epitopes in trachearyparenchyma pit membranes (arrowheads, inset in Fig. 3c). Parenchyma pits showed similar distribution patterns of LM5 and LM6 epitopes in pit membranes with higher amounts of LM5 than LM6 detected (Fig. 8b, c).

Like LM6 epitopes, LM19 (un- and low methyl-esterified HG) and LM20 (low and high methyl-esterified HG) epitopes were observed in the annulus of intertracheid- and
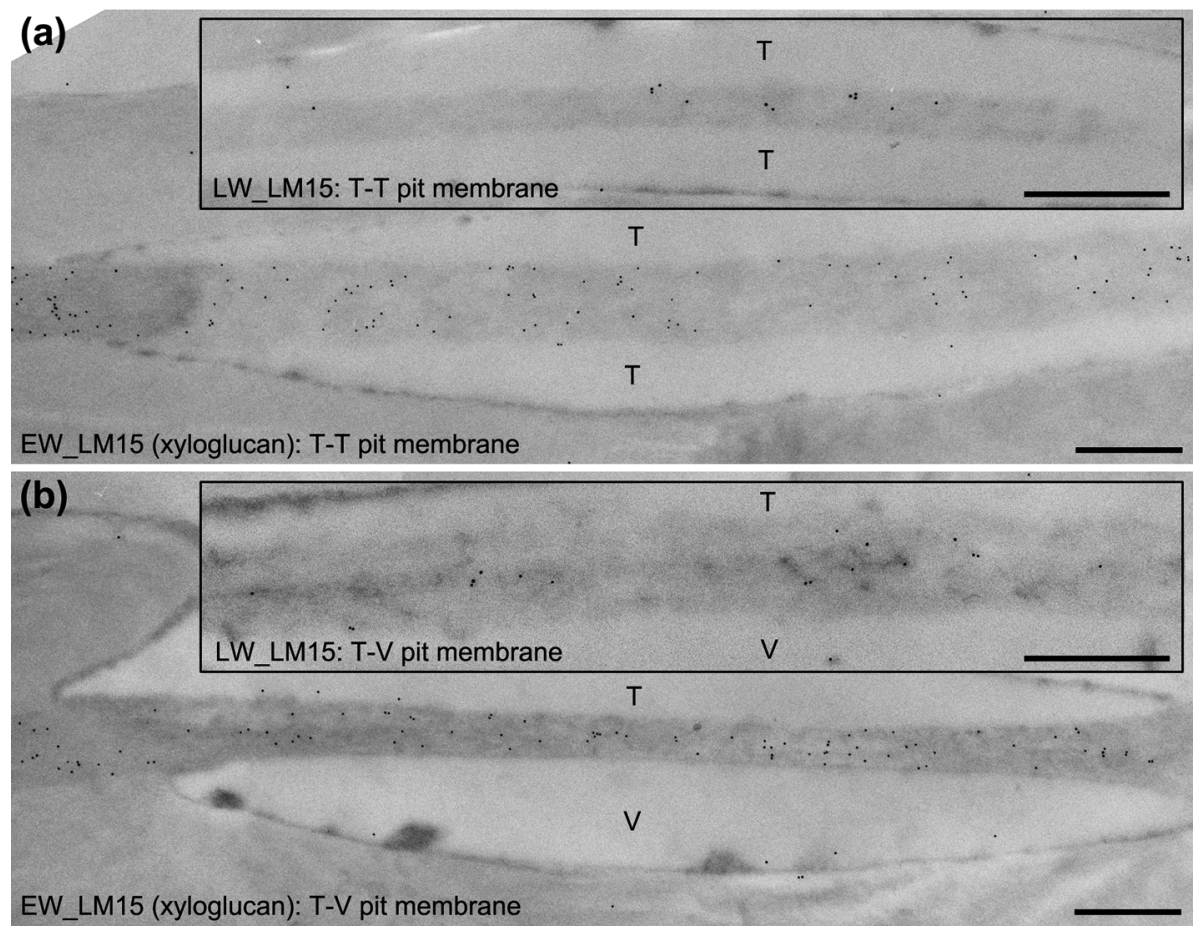

Figure 5. Localization of LM15 (xyloglucan) epitopes in intertracheid (T-T, a) and tracheid-vessel $(\mathrm{T}-\mathrm{V}, \mathrm{b})$ pit membranes of English oak. Note much stronger intensity of xyloglucan epitopes in earlywood (EW; a, b) than in latewood (LW; insets in a, b). - Scale bars $=500 \mathrm{~nm}$. 


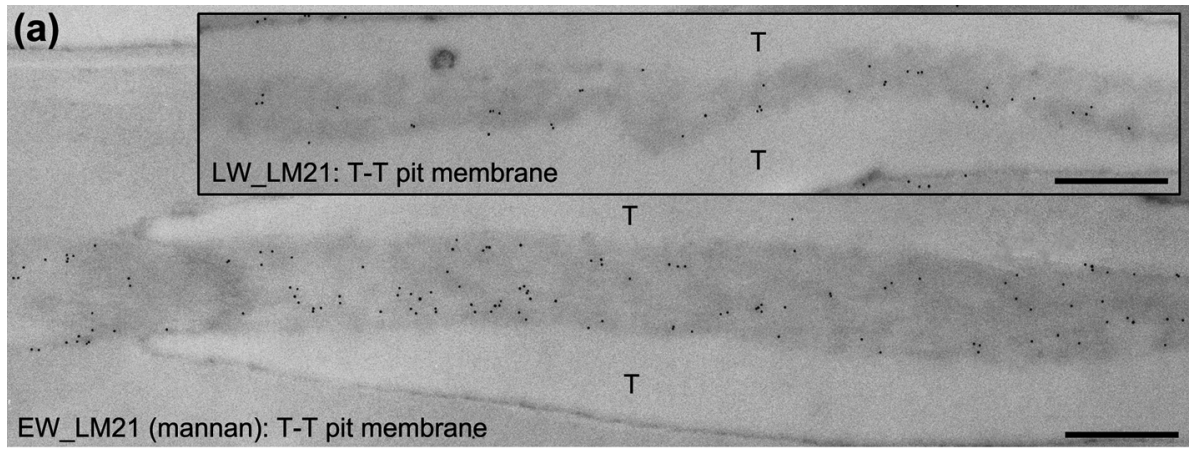

(b)
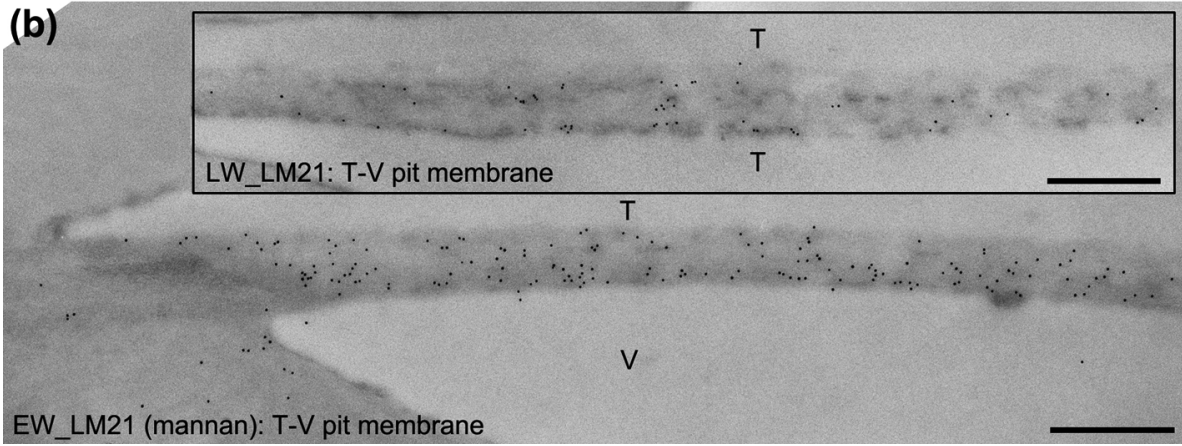

Figure 6. Localization of LM21 epitopes in intertracheid (T-T, a) and tracheid-vessel (T-V, b) pit membranes of English oak. Note stronger intensity of heteromannan epitopes in earlywood $(\mathrm{EW} ; \mathrm{a}, \mathrm{b})$ than in latewood (LW; insets in $\mathrm{a}, \mathrm{b}) .-$ Scale bars $=500 \mathrm{~nm}$.
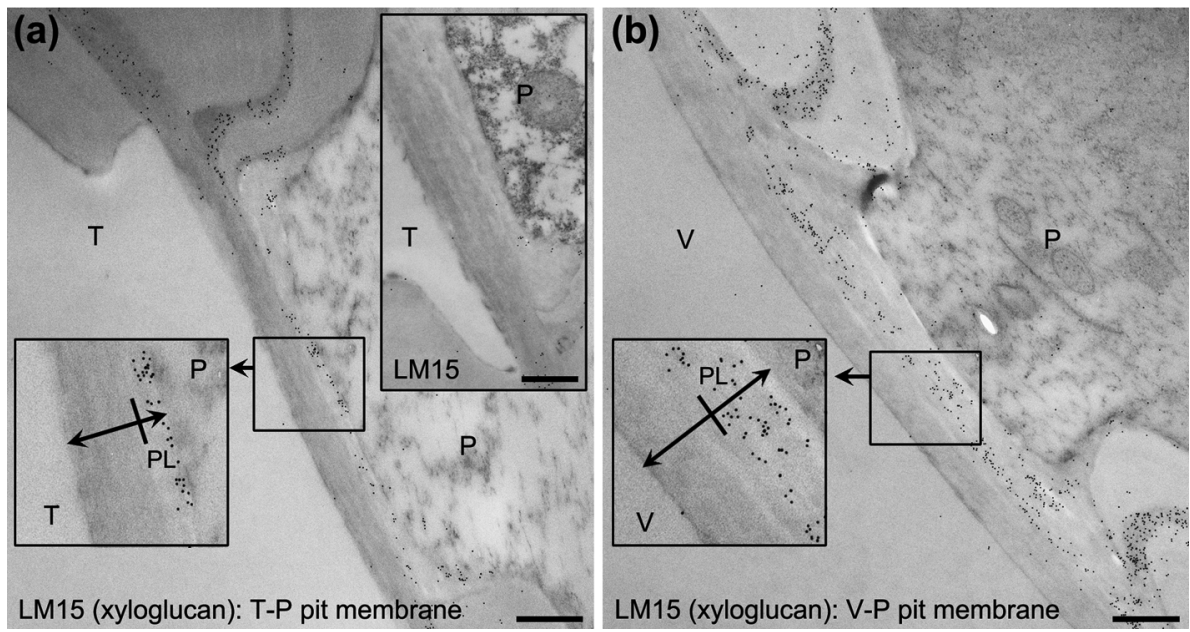

Figure 7. Localization of LM15 (xyloglucan) epitopes in tracheary-parenchyma (T-P/V-P) pit membranes of English oak earlywood. Epitopes were either present $(a, b)$ or absent (inset in a) in T-P and V-P pit membranes. Note distribution of epitopes only in protective layers (PL) of pit membranes $(\mathrm{a}, \mathrm{b})$. - Scale bars $=500 \mathrm{~nm}$. 

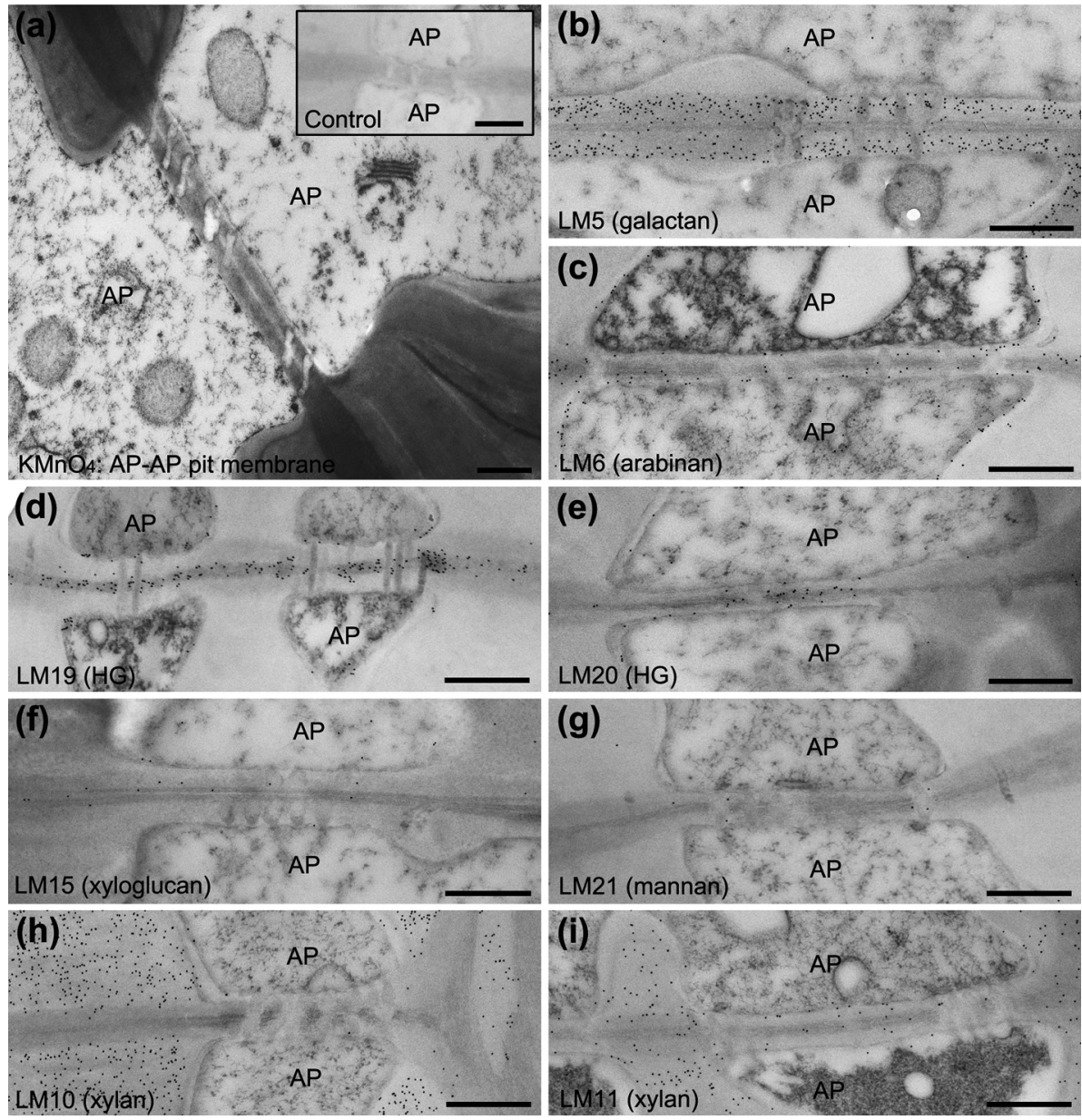

Figure 8. Distribution of phenolic compounds and non-cellulosic polysaccharides in (axial) parenchyma pit membranes (AP) of English oak earlywood. - (a) Some positive $\mathrm{KMnO}_{4}$ staining in pit membranes compared to control (without staining, inset). - (b-e) Distribution of pectin epitopes in pit membranes with stronger intensity of LM5 (b, galactan)/LM19 (d, low methylesterified homogalacturonan, HG) epitopes than in LM6 (c, arabinan)/LM20 (e, high methylesterified HG) epitopes. - (f-i) Localization of hemicellulose epitopes in pit membranes showing presence of sparse LM15 (f, xyloglucan), LM21 (g, heteromannan) and LM11 (i, heteroxylan) epitopes in pit membranes and absence of LM10 (h, heteroxylan) epitopes. - Scale bars $=500 \mathrm{~nm}$.

tracheid-vessel pit membranes (Fig. 4a, b and insets). Both epitopes were absent in the main structural part of pit membranes (Fig. 4a, b and right insets). In trachearyparenchyma pit membranes, abundant LM19 epitopes were only detected in narrow membrane regions facing tracheid and vessel lumina (Fig. 4c, $\mathrm{d}$ and left insets), while LM 20 epitopes were generally abundant across pit membranes (right insets in Fig. 4c, d). LM19 and LM20 epitopes were also observed in parenchyma pit membranes 
with higher amounts of LM19 than LM20 detected (Fig. 8d, e). Consequently, distribution of pectin epitopes was richer in half-bordered (i.e. tracheary-parenchyma pits) and simple (i.e. parenchyma pits) pit membranes than in bordered pit membranes (i.e. intertracheid- and tracheid-vessel pits).

\section{Localization of hemicellulose epitopes}

Figures 5 and 6 show distribution of LM15 (xyloglucan) and LM21 (heteromannan) epitopes respectively in intertracheid- (Fig. 5a, 6a) and tracheid-vessel (Fig. 5b, 6b) pit membranes. Both epitopes were more abundant in EW (Fig. 5, 6) than in LW (insets in Fig. 5, 6). In particular, the intensity of LM15 epitopes in pit membranes was much lower in LW than in EW and varied greatly between pits, including its absence (not shown). In tracheary-parenchyma pit membranes, LM15 epitopes were either abundant (Fig.7a, b) or absent (upper inset in Fig. 7a) in EW and LW. LM15 epitopes were restricted to only protective layers of tracheary-parenchyma pit membranes (bottom inset in Fig. 7a, b). LM 21 epitopes were absent in tracheary-parenchyma pit membranes, irrespective of EW and LW (not shown). No or very few epitopes were detected in parenchyma pit membranes by LM15 and LM21 in both EW (Fig. 8f, g) and LW (not shown). Heteroxylan epitopes (LM10/LM11) were not detected in any of the pit membranes observed except for parenchyma pit membranes that showed sparse presence of LM11 (Fig. 8i) but no LM10 epitopes (Fig. 8h). Together, distribution of hemicellulose epitopes was more pronounced in bordered pit membranes (i.e. intertracheid- and tracheid-vessel pits) than half-bordered (i.e. tracheary-parenchyma pits) and simple (i.e. parenchyma pits) pit membranes.

\section{DISCUSSION}

This study has characterized the chemistry of mature pit membranes in different pit types of English oak xylem using correlated TEM and immunocytochemisty. Although considerable information concerning the chemistry of intervessel pit membranes in hardwoods exists, little information is available about the chemistry of pit membranes associated with imperforate cell elements, particularly vasicentric tracheids. Based on pit membrane chemistry in different pit types observed, some possible physiological relationship between the findings and the function of pit membranes will be discussed in association with water transport in English oak xylem. It is important to remember that this study focused only on samples collected in autumn (i.e. on September 11) and sectors of the second and third growth ring in xylem. We do not exclude possibilities of variations in pit membrane chemistry between growing seasons and between growth rings, particularly from the current (i.e. the first) growth ring which may include a not fully differentiated final latewood zone.

\section{Distribution of phenolic compounds in pit membranes}

With $\mathrm{KMnO}_{4}$ staining, pit membranes of English oak xylem showed great variations in staining patterns between pit types (Table 1). This indicates that distribution of phenolic compounds in pit membranes differs between pit types. Several different types of phenolic compounds, such as lignin and phenolic extractives have been detected in 
pit membranes of hardwoods (Schmitz et al. 2012; Herbette et al. 2015). Unlike EW (all positive), LW showed different patterns of $\mathrm{KMnO}_{4}$ staining in tracheid-vessel pit membranes between vessels (i.e. either negative or positive). Interestingly, Schmitz et al. (2012) reported seasonal variations in patterns of $\mathrm{KMnO}_{4}$ staining in bordered intervessel pit membranes of mangrove woods; negative staining in samples collected in the dry season, positive staining in samples collected in the rainy season and both patterns (i.e. negative and positive) in samples collected between these two periods. These results suggest that variations of $\mathrm{KMnO}_{4}$ staining in tracheid-vessel pit membranes of LW observed may be related to different contributions to water transport between vessels in English oak xylem.

Vessels with positive staining in tracheid-vessel pit membranes may indicate larger contributions than vessels with negative staining to water transport in English oak LW. No variation with positive $\mathrm{KMnO}_{4}$ staining in tracheid-vessel pit membranes of EW may support this hypothesis since water transport in ring porous species like English oak is mainly carried out by the large vessels of EW. No notable staining in intertracheid pit membranes may also reflect smaller contributions in vasicentric tracheids than vessel elements to water transport in English oak xylem. Accumulation of phenolic compounds in pit membranes may allow more active regulation of pit membrane properties associated with water transport in English oak xylem. Based on presence of lignin epitopes in intervessel pit membranes of hybrid poplar, Herbette et al. (2015) proposed that lignin may be related to the ionic effect on hydraulic conductance. It can also be assumed that phenolic compounds including lignin in pit membranes may have effects on air-seeding. Phenolic compounds will make the pit membranes more hydrophobic, thereby resulting in easier penetration of air-water menisci through pores in the cellulose microfibril network.

Phenolic compounds were also detected on the surface of half-bordered trachearyparenchyma pit membranes facing tracheid and vessel lumina. In earlier studies, a similar electron dense layer in vessel-ray parenchyma pit membranes was defined as black cap in peach and dogwood (Wisniewski et al. 1991; Wisniewski \& Davis 1995). Based on labeling with un-/low esterified HG (JIM 5 antibody) similar to labeling by LM19 in this study (Fig. 4c, d), Wisniewski and Davis (1995) proposed that pectins in black cap may form a barrier to water movement and ice propagation at low temperature. However, the biological function of electron dense material accumulated in black cap similar to phenolic compounds in this study was not considered. Simple parenchyma pits also showed presence of phenolic compounds across pit membranes. At present, it is only plausible to suggest that phenolic compounds will presumably affect the permeability of pit membranes in tracheary-parenchyma and inter-parenchyma pits of English oak xylem.

\section{Distribution of pectins and hemicelluloses in bordered pit membranes}

Distribution patterns of pectin epitopes in mature bordered pit membranes were similar between intertracheid- and tracheid-vessel pits in English oak xylem (Table 1). HG and RG-I epitopes were only detected in the annulus of bordered pit membranes. No pectin epitopes using LM5/LM6 and LM19/LM20 were detected in the main 
structural part of bordered pit membranes. These labeling patterns are consistent with previous immunocytochemical studies that reported absence of pectin epitopes in the main structural part of mature bordered intervessel pit membranes (Plavcová \& Hacke 2011; Plavcová et al. 2011; Kim \& Daniel 2013; Herbette et al. 2015). With hemicelluloses, abundant xyloglucan and heteromannan epitopes were detected across mature bordered pit membranes of intertracheid- and tracheid-vessel pits in English oak xylem, particularly in EW. These results differ from recent immunocytochemical studies that showed absence of xyloglucan (LM15) and heteromannan (LM21) epitopes in mature bordered intervessel pit membranes of hybrid poplar (Herbette et al. 2015) and hybrid aspen (Kim \& Daniel 2013). In contrast, results are consistent with immunocytochemical studies that showed distribution of xyloglucan epitopes in mature intervessel pit membranes of European aspen and grapevines (Sun et al. 2011; Kim \& Daniel 2013).

Although the conductive nature of vasicentric tracheids in hardwoods is not fully understood, some studies have suggested that vasicentric tracheids, particularly in oak species may play a role in water transport (Carlquist 1985; Sano et al. 2011). If vasicentric tracheids are significant elements for water transport in English oak xylem like vessels, this study suggests that xyloglucan and heteromannan may play an important role in bordered intertracheid- and tracheid-vessel pit membranes for controlling xylem hydraulic conductance. It has been proposed that like pectins, hemicelluloses can also participate in the swelling/shrinking dynamics of pit membranes depending on ion concentration of xylem (i.e. ion-mediated changes of pit membrane porosity and flow rate) (Li \& Pan 2010; van Doorn et al. 2011). In this respect, it is also expected that the higher intensity of xyloglucan and heteromannan epitopes in bordered pit membranes of EW than of LW may be related to different contributions to water transport between EW and LW bordered pits in English oak xylem, i.e. EW bordered pits have larger contributions than LW bordered pits since major water transport in oak is conducted by EW. Higher concentration of xyloglucan and heteromannan in intertracheid- and tracheid-vessel pit membranes of EW may allow more dynamic changes of pit membrane porosity and flow rate.

Compared to other pit types in English oak xylem studied, the chemistry of bordered pit membranes (i.e. intertracheid- and tracheid-vessel pits) differed (Table 1). Overall, bordered pit membranes were poorer and richer in distribution of pectin and hemicellulose epitopes than half-bordered and simple pit membranes, respectively. Bordered pit membranes showed absence of HG and RG-I epitopes (except for annulus) but showed presence of heteromannan epitopes. In contrast, half-bordered (between tracheary-parenchyma pits) and simple pit membranes (interparenchyma pits) (see below) showed opposite patterns (i.e. presence of HG and RG-I but absence of heteromannan epitopes). Distribution patterns of xyloglucan epitopes in bordered pit membranes also differed from other pit types, particularly in EW. Xyloglucan epitopes were detected across bordered pit membranes, whereas they were only detected in protective layers of half-bordered pit membranes. Xyloglucan epitopes were also almost absent in simple pit membranes. Since bordered pits are cavities in major conduits (i.e. vasicentric tracheids and vessels) of English oak xylem, these results may also reflect a specific 
biological role of heteromannan and xyloglucan in bordered pit membranes in relation to regulation of water transport within English oak xylem.

\section{Distribution of pectins and hemicelluloses in half-bordered pit membranes}

HG and RG-I epitopes were detected across half-bordered pit membranes of tracheary-parenchyma pits in English oak xylem (Table 1). Similar results were also observed in half-bordered vessel-ray pit membranes of several hardwood species (Rioux et al. 1998; Plavcová \& Hacke 2011; Kim \& Daniel 2013). By differences in labeling intensity between LM5 (galactan, no/sparse labeling) and LM6 (arabinan, abundant labeling) antibodies (Fig. 3c, d), it can be expected that RG-I in half-bordered pit membranes of English oak may be highly substituted with arabinan. With HG epitopes, half-bordered tracheary-parenchyma pit membranes showed spatial differences in distribution depending on antibody types (Fig. 4c, d). LM19 epitopes (indicating un- and low methylesterified $\mathrm{HG}$ ) were only detected in narrow regions of membranes close to tracheid and vessel lumina, whereas LM20 (indicating low and highly methyl-esterified HG) were detected across membranes. These results indicate heterogeneity in the degree of HG esterification between pit membrane regions, i.e. membranes close to tracheid and vessel lumina are composed of low methyl-esterified HG, whereas other membrane regions are composed of highly methyl-esterified HG. Concerning hemicelluloses, only xyloglucan (LM15) epitopes were detected in half-bordered pit membranes. No heteromannan and heteroxylan epitopes were detected in half-bordered pit membranes of English oak. These results differ from half-bordered vessel-ray pit membranes of hybrid aspen that showed presence of heteroxylan epitopes as well as xyloglucan epitopes in protective layers (Kim et al. 2012; Kim \& Daniel 2013). This may indicate chemical variations of half-bordered pit membranes, particularly in protective layer regions between hardwood species. Based on restricted distribution of xyloglucan epitopes only in protective layers and together with heterogeneity in the degree of HG esterification between pit membrane regions as outlined above, it can be expected that half-bordered tracheary-parenchyma pit membranes of English oak xylem may be composed primarily of several distinct chemical domains (Table 1). Similar chemical heterogeneity between pit membrane regions has also been reported in half-bordered vessel-ray pits of two poplar species (Kim \& Daniel 2013) and cross-field pits of pine and spruce (softwoods, Kim \& Daniel 2014). The reason for variations in xyloglucan epitopes (i.e. presence and absence) between half-bordered pit membranes of English oak xylem is currently unclear.

Considering the roles of half-bordered tracheary-parenchyma pit membranes associated with water transport in living English oak xylem, very little is known. However, on the basis of previous studies that propose solutes released from living cells (i.e. parenchyma cells) to vessels influence water transport in xylem (Braun 1984; De Boer \& Volkov 2003; Nardini et al. 2011), we may assume that pectins and xyloglucan in half-bordered pit membranes may play a role for controlling the amount of ions (i.e. ionic concentrations) in xylem conduits, thereby influencing ultimately ion-mediated changes of xylem hydraulic conductance in English oak. Wisniewski et al. (1987) showed permeability of half-bordered vessel-parenchyma pit membranes using lanthanum staining (penetrate voids as small as $2 \mathrm{~nm}$ ) in some hardwood species. 


\section{Distribution of pectins and hemicelluloses in simple pit membranes}

Like half-bordered pit membranes of tracheary-parenchyma pits, abundant HG and RG-I epitopes were detected in simple pit membranes of parenchyma pits (Table 1). By differences in labeling intensity between antibodies (i.e. LM19/LM20 for HG, LM5/ LM6 for RG-I, Fig. 8b-e), it can be expected that parenchyma pit membranes may contain high levels of low methyl-esterified HG (LM19) and RG-I substituted with galactan (LM5). Similar distribution patterns of HG were also detected in simple ray pit membranes (between two ray parenchyma cells) of hybrid aspen (Kim et al.2012). Like English oak (a hardwood) in this study, different labeling intensity of HG and RG-I epitopes between antibodies were also observed in simple ray pit membranes of Scots pine and Norway spruce seedlings (Kim \& Daniel 2014). However, distribution patterns of each antibody differed from English oak, particularly RG-I. Scots pine and Norway spruce showed higher levels of RG-I substituted with arabinan (LM6) than with galactan (LM5) (i.e. in contrast to English oak).

\section{CONCLUSIONS}

This study extends our knowledge of pit membrane chemistry in hardwoods. In particular, we show the presence of hemicelluloses (heteromannan and xyloglucan) in mature bordered pit membranes of English oak xylem (i.e. intertracheid- and tracheid-vessel pits). These results suggest that heteromannan and xyloglucan may be able to play an important role in changes of bordered pit membrane properties in English oak xylem similar to pectin based ion-mediated changes of pit membrane porosity. Results also demonstrate that there are great variations in pit membrane chemistry in relation to non-cellulosic polysaccharides between pit types in English oak xylem. Overall, bordered pit membranes are rich in hemicellulose epitopes, whereas half-bordered (i.e. tracheary-parenchyma pits) and simple (i.e. interparenchyma pits) pit membranes are rich in pectin epitopes. The distribution of phenolic compounds differs between pit types and between EW and LW in English oak. Altogether, the chemistry of pit membranes and the variations between pit types observed may be closely linked to the different biological functions of pit types for fine regulation of water transport in English oak xylem.

\section{ACKNOWLEDGEMENTS}

The present work was supported by Formas projects 2008-1399, 2009-582, 2011-416, and 2015-469.

\section{REFERENCES}

Braun HJ. 1984. The significance of the accessory tissues of the hydrosystem for osmotic water shifting as the second principle of water ascent, with some thoughts concerning the evolution of trees. IAWA Bull. n.s. 5: 275-294.

Carlquist S. 1985. Vasicentric tracheids as a drought survival mechanism in the woody flora of southern California and similar regions; review of vasicentric tracheids. Aliso 11: 37-68.

Choat B, Cobb AR \& Jansen S. 2008. Structure and function of bordered pits: new discoveries and impacts on whole-plant hydraulic function. New Phytol. 177: 608-626. 
De Boer AH \& Volkov V. 2003. Logistics of water and salt transport through the plant: structure and functioning of the xylem. Plant Cell Environ. 26: 87-101.

Gortan E, Nardini A, Salleo S \& Jansen S. 2011. Pit membrane chemistry influences the magnitude of ion-mediated enhancement of xylem hydraulic conductance in four Lauraceae species. Tree Physiol. 31: 48-58.

Herbette S, Bouchet B, Brunel N, Bonnin E, Cochard H \& Guillon F. 2015. Immunolabelling of intervessel pits for polysaccharides and phenolics helps in understanding their hydraulic properties in Populus tremula $\times$ alba. Ann. Bot. 115: 187-199.

InsideWood. 2004-onwards. Published on the internet (http://insidewood.lib.ncsu.edu/search).

Jansen S, Choat B, Vinckier S, Lens F, Schols P \& Smets E. 2004. Intervascular pit membranes with a torus in the wood of Ulmus (Ulmaceae) and related genera. New Phytol. 163: $51-59$.

Jones L, Seymour GB \& Knox JP. 1997. Localization of pectic galactan in tomato cell walls using a monoclonal antibody specific to $(1 \rightarrow 4)-\beta$-D-galactan. Plant Physiol. 113: 1405-1412.

Kim JS \& Daniel G. 2013. Developmental localization of homogalacturonan and xyloglucan epitopes in pit membranes varies between pit types in two poplar species. IAWA J. 34: $245-262$.

Kim JS \& Daniel G. 2014. Distributional variation of lignin and non-cellulosic polysaccharide epitopes in different pit membranes of Scots pine and Norway spruce seedlings. IAWA J. 35: 407-429.

Kim JS, Sandquist D, Sundberg B \& Daniel G. 2012. Spatial and temporal variability of xylan distribution in differentiating secondary xylem of hybrid aspen. Planta 235: 1315-1330.

Li S, Lens F, Espino S, Karimi Z, Klepsch M, Schenk HJ, Schmitt M, Schuldt B \& Jansen S. 2016. Intervessel pit membrane thickness as a key determinant of embolism resistance in angiosperm xylem. IAWA J. 37: 152-171.

Li X \& Pan X. 2010. Hydrogels based on hemicellulose and lignin from lignocelluloses biorefinery: a mini-review. J. Biobased Mater. Bio. 4: 289-297.

Marcus S, Blake AW, Benians TAS, Lee KJD, Poyser C, Donaldson L, Leroux O, Rogowski A, Petersen HL, Boraston A, Gilbert HJ, Willats WGT \& Knox JP. 2010. Restricted access of proteins to mannan polysaccharides in intact plant cell walls. Plant J. 64: 191-203.

Marcus SE, Verhertbruggen Y, Hervè C, Ordaz-Ortiz J, Farkas V, Pedersen HL, Willats WGT \& Knox JP. 2008. Pectic homogalactronan masks abundant sets of xyloglucan epitopes in plant cell walls. BMC Plant Biol. 8: 60.

McCartney L, Marcus SE \& Knox JP. 2005. Monoclonal antibodies to plant cell wall xylans and arabinoxylans. J. Histochem. Cytochem. 53: 543-546.

Nardini A, Salleo S \& Jansen S. 2011. More than just a vulnerable pipeline: xylem physiology in the light of ion-mediated regulation of plant water transport. J. Exp. Bot. 62: 4701-4718.

Plavcová L \& Hacke UG. 2011. Heterogeneous distribution of pectin epitopes and calcium in different pit types of four hardwood species. New Phytol. 192: 885-897.

Plavcová L, Hacke UG \& Sperry JS. 2011. Linking irradiance-induced changes in pit membrane ultrastructure with xylem vulnerability to cavitation. Plant Cell Environ. 34: 501-513.

Rioux D, Nicole M, Simard M \& Ouellette GB. 1998. Immunocytochemical evidence that secretion of pectin occurs during gel (gum) and tylosis formation in trees. Phytopathology 88: 494-504.

Sano Y \& Jansen S. 2006. Perforated pit membranes in imperforate tracheary elements of some hardwoods. Ann. Bot. 97: 1045-1053.

Sano Y, Morris H, Shimada H, Ronse De Craene LP \& Jansen S. 2011. Anatomical features associated with water transport in imperforate tracheary elements of vessel-bearing hardwoods. Ann. Bot. 107: 953-964. 
Schenk HJ, Steppe K \& Jansen S. 2015. Nanobubbles: a new paradigm for air-seeding in xylem. Trends Plant Sci. 20: 199-205.

Schmitz N, Koch G, Beeckman H, Koedam N, Robert EMR \& Schmitt U. 2012. A structural and compositional analysis of intervessel pit membranes in the sapwood of some mangrove woods. IAWA J. 33: 243-256.

Schweingruber FH. 1990. Anatomy of European woods: an atlas for the identification of European trees, shrubs and dwaft shrubs. Paul Haupt, Bern, Stuttgart.

Sun Q, Greve LC \& Labavitch JM. 2011. Polysaccharide compositions of intervessel pit membranes contribute to Pierce's disease resistance of grapevines. Plant Physiol. 155: 1976-1987.

van Doorn WG, Hiemstra T \& Fanourakis D. 2011. Hydrogel regulation of xylem water flow: an alternative hypothesis. Plant Physiol. 157: 1642-1649.

Verhertbruggen Y, Marcus SE, Haeger A, Ordaz-Ortiz JJ \& Knox JP. 2009. An extended set of monoclonal antibodies to pectic homogalacturonan. Carbohydr. Res. 344: 1858-1862.

Willats WGT, Marcus SE \& Knox JP. 1998. Generation of a monoclonal antibody specific to $(1 \rightarrow 5)-\alpha$-L-arabinan. Carbohydr. Res. 308: 149-152.

Wisniewski M, Ashworth E \& Schaffer K. 1987. The use of lanthanum to characterize cell wall permeability in relation to deep supercooling and extracellular freezing in woody plants. Protoplasma 139: 105-116.

Wisniewski M \& Davis G. 1995. Immunogold localization of pectins and glycoproteins in tissues of peach with reference to deep supercooling. Trees 9: 253-260.

Wisniewski M, Davis G \& Schaffer K. 1991. Mediation of deep supercooling of peach and dogwood by enzymatic modifications in cell-wall structure. Planta 184: 254-260.

Zwieniecki MA, Melcher PJ \& Holbrook NM. 2001. Hydrogel control of xylem hydraulic resistance in plants. Science 291: 1059-1061.

Accepted: 4 April 2016

Associate Editor: Lloyd A. Donaldson 